\title{
HARNACK INEQUALITY AND REGULARITY OF $p$-LAPLACE EQUATION ON COMPLETE MANIFOLDS
}

\author{
XI ZHANG
}

\begin{abstract}
In this paper, we will derive a mean value inequality and a Harnack inequality for nonnegative functions which satisfies the differential inequality

$$
\left|\operatorname{div}\left(|f|^{p-2} \nabla f\right)\right| \leq A \quad f^{p-1}
$$

in the weak sence on complete manifolds, where constants $A \geq 0, p>1$; as a consequence, we give a $C^{\alpha}$ estımate for weak solutions of the above differential inequality, then we generalize the results in [1], [2].

We would thank Professor Z. G. Bai and Professor Y B. Shen for their long time encourgement, we also thank the referee for invaluable comments.
\end{abstract}

\section{Introduction}

Let $M$ be a complete Riemannian manfold, and $f$ be a real $C^{2}$ function on $M$. Fix $p>1$ and consider a compact domain $\Omega \subset M$. The $p$-energy of $f$ on $\Omega$, is defined to be,

$$
E_{p}(\Omega, f)=\frac{1}{p} \int_{\Omega}|\nabla F|^{p} d v_{g}
$$

The function $f$ is said to be $p$-harmonic on $M$ if $f$ is a critical point of $E_{p}(\Omega, *)$ for every compact domain $\Omega \subset M$. Equaivalently, $f$ satisfies the EulerLagrange equation.

$$
\Delta_{p} f \equiv \operatorname{div}\left(|\nabla f|^{p-2} \nabla f\right)=0
$$

Let $g \in H_{1, p}(\Omega)$ satisfies the equation (1.2) in the weak sence, it is:

$$
\int_{\Omega}\left\langle|\nabla g|^{p-2} \cdot \nabla_{g}, \nabla \phi\right\rangle d v_{g}=0
$$

for any $\phi \in C_{0}^{\infty}(\Omega)$, then $g$ is said to be a weakly solution of eguation (1.2) on $\Omega$.

Key words and phrases: Complete manifold, p-Laplace Operator, Poincaré inequality, Hölder contınuouty, Moser's iteration.

Received July 5, 1999; revised November 1, 1999. 
Definition. $f$ is called a weakly $p$-harmonic if $f \in H_{1, p}^{\text {loc }}(M)$ is a weak solution of the Euler-Lagrange equation of the $p$-energy functional (1.2) as follows:

$$
\int_{M}|f|^{p-2}\langle d \eta, d f\rangle=0
$$

for all $\eta \in C_{0}^{\infty}(M)$.

Regularity estimates for elliptic systems on domain $\Omega \subset R^{n}$, in particular the Euler-Lagrange equation for $p$-energy, were first obtained by Uhlenbeck [3] for $p \geq 2$, and later by Tolksdorff [4] for $p>1$. The aim of this paper is to obtain regularity estimates for a more general class of equations on complete manifolds. In section two and section three, by using the iteration procedure of Moser and discussing like that in [1], we derive a mean value inequality and a Harnack inequality for nonnegative functions which satisfies the differetial inequality of the following form:

$$
\left|\operatorname{div}\left(|f|^{p-2} \nabla f\right)\right| \leq A \cdot f^{p-1}
$$

in the weak sence for some constant $A \geq 0$. As a special case: $A=0$, using the above Harnack inequality, we can derive a (global) Harnack inequality for weakly $p$-harmonic function which is similar to a result of M. Rigoli, M. Salvatori, and M. Vignati [2]. At the end of this paper, we will give a $C^{\alpha}$ etimate for solutions of above differential inequality. When $p=2$, the above mean value inequality, Harnack inequality, and $C^{\alpha}$ estimate is just the results due to $\mathrm{P}$. $\mathrm{Li}$ in [1]. On the other hand, using the above Harnack inequality, we can obtain a Liouville type theorem which can be see a generalization of the result in [2].

THEOREM. Let $M$ be a complete noncompact Riemannian manifold with nonnegative Ricci curvature. Then there exists a constant $0<\alpha \leq 1$ such that any p-harmonic function $f$ defined on $M$ satisfying the growth condition

$$
|f(x)|=\circ\left(\rho^{\alpha}(x)\right)
$$

as $x \rightarrow 0$, where $\rho(x)$ denotes the geodesic distance from o to $x$; must be identically constant.

\section{Mean-value inequality}

LEMMA 2.1. Let $M$ be a complete Riemannian manifold, and geodesic ball $B_{o}(R)$ satisfies: $B_{o}(R) \cap \partial M=\emptyset$. If $f \in H_{1, p}\left(B_{o}(R)\right)$ is a nonnegative function, and satisfies the following inequality in weak sence:

$$
\operatorname{div}\left(|\nabla f|^{p-2} \nabla f\right) \geq-A \cdot f^{p-1}
$$

where constants $A \geq 0 ; p>1$, then for any $0<r \leq R, \tilde{q} \geq p$ and nonnegative function $\eta \in C_{0}^{\infty}\left(B_{o}(r)\right)$, there: 


$$
\begin{aligned}
\int_{B_{o}(r)} \eta^{p} \cdot f^{\tilde{q}-p} \cdot|\nabla f|^{p} \leq & \frac{2^{p} \cdot(p-1)^{p-1}}{(\tilde{q}-p+1)^{p}} \cdot \int_{B_{o}(r)} f^{\tilde{q}} \cdot|\nabla \eta|^{p} \\
& +\frac{2 A}{\tilde{q}-p+1} \cdot \int_{B_{o}(r)} \eta^{p} \cdot f^{\tilde{q}}
\end{aligned}
$$

Proof. Multiplying $\eta^{p} \cdot f^{\tilde{q}-p+1}$ to (2.1), and integrating yields

$$
\int_{B_{o}(r)} \eta^{p} \cdot f^{\tilde{q}-p+1} \cdot \operatorname{div}\left(|\nabla f|^{p-2} \nabla f\right) \geq-A \cdot \int_{B_{o}(r)} \eta^{p} \cdot f^{\tilde{q}}
$$

Using Green's formula; Schwartz inequality; and Young inequality, we have:

$$
\begin{aligned}
(\tilde{q}-p+1) & \cdot \int_{B_{o}(r)} \eta^{p} \cdot f^{\tilde{q}-p} \cdot|\nabla f|^{p} \\
& \leq A \cdot \int_{B_{o}(r)} \eta^{p} \cdot f^{\tilde{q}}-p \cdot \int_{B_{o}(r)}|\nabla f|^{p-2} \cdot f^{\tilde{q}-p+1} \cdot \eta^{p-1} \cdot\langle\nabla f, \nabla \eta\rangle \\
& \leq A \cdot \int_{B_{o}(r)} \eta^{p} \cdot f^{\tilde{q}}+p \cdot \int_{B_{o}(r)}|\nabla f|^{p-1} \cdot f^{\tilde{q}-p+1} \cdot \eta^{p-1} \cdot|\nabla \eta| \\
& \leq A \cdot \int_{B_{o}(r)} \eta^{p} \cdot f^{\tilde{q}}+\frac{\tilde{q}-p+1}{2} \cdot \int_{B_{o}(r)}|\nabla f|^{p} \cdot f^{\tilde{q}-p} \cdot \eta^{p} \\
& +\left[\frac{2 p-2}{\tilde{q}-p+1}\right]^{p-1} \int_{B_{o}(r)} f^{\tilde{q}} \cdot|\nabla \eta|^{p}
\end{aligned}
$$

then

$$
\begin{aligned}
\int_{B_{o}(r)} \eta^{p} \cdot f^{\tilde{q}-p} \cdot|\nabla f|^{p} \leq \frac{2^{p} \cdot(p-1)^{p-1}}{(\tilde{q}-p+1)^{p}} \int_{B_{o}(r)} f^{\tilde{q}} \cdot|\nabla \eta|^{p} \\
+\frac{2 A}{\tilde{q}-p+1} \cdot \int_{B_{o}(r)} \eta^{p} \cdot f^{\tilde{q}}
\end{aligned}
$$

Proposition 2.2. Let $M$ be a complete Riemannian manifold, and a geodesic ball $B_{o}(R)$ satisfies: $B_{o}(R) \cap \partial M=\emptyset$. If there exists a sobolev inequality of the following form:

$$
\begin{aligned}
\left(\int_{B_{o}(r)} \phi^{p \mu /(\mu-p)}\right)^{(\mu-p) / p \mu} \leq & C_{s} \cdot V\left(B_{o}(r)\right)^{-1 / \mu} \cdot r \\
& \cdot\left\{\left(\int_{B_{o}(r)}|\nabla \phi|^{p}\right)^{1 / p}+r^{-1} \cdot\left(\int_{B_{o}(r)}|\phi|^{p}\right)^{1 / p}\right\}
\end{aligned}
$$


for any $\phi \in H_{1, p}^{c}\left(B_{o}(r)\right)$ and $0<r<R$. Where constants $\mu>p, C_{s}>0$, and $V\left(B_{o}(r)\right)$ denotes the volume of geodesic ball $B_{o}(r)$. Assuming $f \in H_{1, p}\left(B_{o}(R)\right)$ is a nonnegative function, and satisfies the following inequality in weak sence,

$$
\operatorname{div}\left(|\nabla f|^{p-2} \nabla f\right) \geq-A \cdot f^{p-1}
$$

for some constant $A \geq 0$; then for any $q>0,0<\theta<1$, and $0<r<R$; there must exist a constant $C_{1}>0$, depending only on $q, \mu, C_{s}, p$, such that:

$$
\sup _{B_{o}(\theta r)} f \leq C_{1} \cdot\left(A r^{P}+(1-\theta)^{-p}\right)^{\mu \cdot(q+p) /(p \cdot q)} \cdot V\left(B_{o}(r)\right)^{-1 / q} \cdot\left(\int_{B_{o}(r)} f^{q}\right)^{1 / q}
$$

Proof. Setting $0<r_{1}<r_{2} \leq r, \tilde{q} \geq p$, and let $\eta \in C_{0}^{\infty}\left(B_{o}(R)\right)$ be the cut-off function

$$
\eta(x)= \begin{cases}1 ; & x \in B_{o}\left(r_{1}\right) \\ 0 ; & x \in B_{o}(R) \backslash B_{o}\left(r_{2}\right)\end{cases}
$$

$\eta(x) \in[0,1],|\nabla \eta| \leq 2 /\left(r_{2}-r_{1}\right)$. Using the sobolev inequality (2.3) and CauchySchwartz inequality, we have

$$
\begin{aligned}
& \left(\int_{B_{o}\left(r_{1}\right)} f^{\tilde{q} \cdot \mu /(\mu-p)}\right)^{(\mu-p) / p \mu} \\
& \leq\left(\int_{B_{o}\left(r_{2}\right)}\left(\eta \cdot f^{\tilde{q} / p}\right)^{p \cdot \mu /(\mu-p)}\right)^{(\mu-p) / p \mu}=\left(\int_{B_{o}(r)}\left(\eta \cdot f^{\tilde{q} / p}\right)^{p \cdot \mu /(\mu-p)}\right)^{(\mu-p) / p \mu} \\
& \leq C_{s} \cdot V\left(B_{o}(r)\right)^{-1 / \mu} \cdot\left[r\left(\int_{B_{o}(r)}\left|\nabla\left(\eta \cdot f^{\tilde{q} / p}\right)\right|^{p}\right)^{1 / p}+\left(\int_{B_{o}(r)} \eta^{p} \cdot f^{\tilde{q}}\right)^{1 / p}\right] \\
& \leq C_{s} \cdot V\left(B_{o}(r)\right)^{-1 / \mu} \cdot\left[\left(\int_{B_{o}(r)} \eta^{p} \cdot f^{\tilde{q}}\right)^{1 / p}\right] \\
& \left.+2 r\left(\int_{B_{o}(r)}\left(|\nabla \eta|^{p} \cdot f^{\tilde{q}}+\left(\frac{\tilde{q}}{p}\right)^{p} \cdot \eta^{p} \cdot f^{\tilde{q}-p} \cdot|\nabla f|^{p}\right)\right)^{1 / p}\right]
\end{aligned}
$$

by formula (2.2), we have: 


$$
\begin{aligned}
\left(\int_{B_{o}\left(r_{1}\right)} f^{\tilde{q} \cdot \mu /(\mu-p)}\right)^{(\mu-p) / p \mu} & \\
\leq & C_{s} \cdot V\left(B_{o}(r)\right)^{-1 / \mu} \cdot\left[\left(\int_{B_{o}(r)} \eta^{p} \cdot f^{\tilde{q}}\right)^{1 / p}\right. \\
& +2 r\left(\int_{B_{o}(r)}|\nabla \eta|^{p} \cdot f^{\tilde{q}}+\left(\frac{\tilde{q}}{p}\right)^{p} \cdot \frac{2^{p} \cdot(p-1)^{p-1}}{(\tilde{q}-p+1)^{p}} \cdot \int_{B_{o}(r)} f^{\tilde{q}} \cdot|\nabla \eta|^{p}\right. \\
& \left.\left.+\left(\frac{\tilde{q}}{p}\right)^{p} \cdot \frac{2 A}{\tilde{q}-p+1} \cdot \int_{B_{o}(r)} \eta^{p} \cdot f^{\tilde{q}}\right)^{1 / p}\right] \\
\leq & C_{s} \cdot V\left(B_{o}(r)\right)^{-1 / \mu} \cdot\left[16 \tilde{q} \cdot\left(A r^{p}+\frac{r^{p}}{\left(r_{2}-r_{1}\right)^{p}}\right)^{1 / p}+1\right] \cdot\left(\int_{B_{o}\left(r_{2}\right)} f^{\tilde{q}}\right)^{1 / p} \\
\leq & 17 \cdot \tilde{q} \cdot C_{s} \cdot V\left(B_{o}(r)\right)^{-1 / \mu} \cdot\left(A r^{p}+\frac{r^{p}}{\left(r_{2}-r_{1}\right)^{p}}\right)^{1 / p} \cdot\left(\int_{B_{o}\left(r_{2}\right)} f^{\tilde{q}}\right)^{1 / p}
\end{aligned}
$$

Let:

$$
\left\{\begin{array}{l}
R_{l}=r_{3}+\left(r_{4}-r_{3}\right) \cdot 2^{-l} \\
q_{i}=p \cdot\left(\frac{\mu}{\mu-p}\right)^{l}
\end{array}\right.
$$

where $0<r_{3}<r_{4} \leq r$. Denote $k=\mu /(\mu-p)$, appling (2.5) to $r_{1}=R_{l+1}, r_{2}=$ $R_{l}, \tilde{q}=q_{i}$, we have:

$$
\begin{aligned}
\left\{\int_{B_{o}\left(R_{i+1}\right)} f^{p \cdot k^{i+1}}\right\}^{k^{-(i+1)}} & \left(17 \cdot C_{s} \cdot V\left(B_{o}(r)\right)^{-1 / \mu}\right)^{p / k^{l}} \cdot\left(p \cdot k^{l}\right)^{p / k^{l}} \\
\leq & \left(A r^{p}+\frac{r^{p}}{\left(r_{4}-r_{3}\right)^{p}}\right)^{1 / k^{l}} \cdot 2^{(i+1) p / k^{l}} \cdot\left\{\int_{B_{o}\left(R_{l}\right)} f^{p \cdot k^{l}}\right\}^{k^{-l}}
\end{aligned}
$$

Observe that $\lim _{i \rightarrow \infty} R_{l}=r_{3}$, and iterating the inequality (2.6), we conclude that:

$$
\sup _{B_{o}\left(r_{s}\right)} f^{p} \leq C_{2} \cdot\left(A r^{p}+\frac{r^{p}}{\left(r_{4}-r_{3}\right)^{p}}\right)^{\mu / p} \cdot V\left(B_{o}(r)\right)^{-1} \cdot \int_{B_{o}\left(r_{4}\right)} f^{p}
$$

where we have used $\sum_{l=0}^{\infty} 1 / k^{l}=\mu / p, \sum_{l=0}^{\infty}(i+1) / k^{l}=\mu^{2} / p^{2}$, and denote $C_{2}=$ $\left(17 \cdot p \cdot C_{s} / k\right)^{\mu} \cdot(2 k)^{\mu^{2} / p}$. 
(a) When $q \geq p$, appling (2.7) to $r_{3}=\theta \cdot R$, and $r_{4}=R$, by Hölder inequality, we have:

$$
\begin{aligned}
\sup _{B_{o}(\theta r)} f & \leq C_{2}^{1 / p} \cdot\left(A r^{p}+(1-\theta)^{-p}\right)^{\mu / p^{2}} \cdot\left(\frac{\int_{B_{o}(r)} f^{p}}{V\left(B_{o}(r)\right)}\right)^{1 / p} \\
& \leq C_{2}^{1 / p} \cdot\left(A r^{p}+(1-\theta)^{-p}\right)^{\mu / p^{2}} \cdot\left(\frac{\int_{B_{o}(r)} f^{q}}{V\left(B_{o}(r)\right)}\right)^{1 / q}
\end{aligned}
$$

(b) When $0<q<p$. Let $h_{0}=\theta r, h_{1}=\theta r+2^{-1} \cdot(1-\theta) \cdot r_{1}, \ldots, h_{i}=h_{i-1}+$ $2^{-l} \cdot(1-\theta) \cdot r$, for each $i=1,2,3, \ldots$; applying $(2.7)$ to $r_{3}=h_{i}, r_{4}=h_{i+1}$, we have:

$$
\begin{aligned}
\sup _{B_{o}\left(h_{t}\right)} f^{p} \leq & C_{2} \cdot\left(A r^{p}+\frac{r^{p}}{\left(h_{i+1}-h_{i}\right)^{p}}\right)^{\mu / p} \cdot V\left(B_{o}(r)\right)^{-1} \cdot \int_{B_{o}\left(h_{i+1}\right)} f^{p} \\
\leq & C_{2} \cdot\left(A r^{p}+(1-\theta)^{-p}\right)^{\mu / p} \cdot 2^{(i+1) \mu} \cdot V\left(B_{o}(r)\right)^{-1} \\
& \cdot \int_{B_{o}\left(h_{l+1}\right)} f^{q} \cdot \sup _{B_{o}\left(h_{i+1}\right)} f^{p-q}
\end{aligned}
$$

denote $M(i)=\sup _{B_{o}\left(h_{t}\right)} f^{p},(2.9)$ becomes:

$$
\begin{aligned}
M(i) \leq & C_{2} \cdot\left(A r^{p}+(1-\theta)^{-p}\right)^{\mu / p} \cdot 2^{(i+1) \mu} \cdot V\left(B_{o}(r)\right)^{-1} \\
& \cdot \int_{B_{o}(r)} f^{q} \cdot M(1+i)^{1-(q / p)}
\end{aligned}
$$

Let $\lambda=1-(q / p)$, interating the inequality, we conclude that:

$$
\begin{aligned}
M(0) \leq & \prod_{l=0}^{j-1}\left\{C_{2} \cdot\left(A r^{p}+(1-\theta)^{-p}\right)^{\mu / p} \cdot V\left(B_{o}(r)\right)^{-1} \cdot \int_{B_{o}(r)} f^{q}\right\}^{\lambda^{l}} \\
& \cdot 2^{(i+1) \mu \lambda^{l}} \cdot M(j)^{\lambda^{\prime}}
\end{aligned}
$$

let $j \rightarrow+\infty$, we have

$$
\begin{aligned}
\sup _{B_{o}(\theta r)} f \leq & \left\{C_{2} \cdot 2^{\mu \cdot p^{2} q^{-1}}\left(A r^{p}+(1-\theta)^{-p}\right)^{\mu / p}\right. \\
& \left.\cdot V\left(B_{o}(r)\right)^{-1}\right\} q^{-1} \cdot\left(\int_{B_{o}(r)} f^{q}\right)^{1 / q}
\end{aligned}
$$

In any event, (2.8), (2.12) imply that, for any $q>0$, we have the inequality

$$
\sup _{B_{o}(\theta r)} f \leq C_{1} \cdot\left(A r^{P}+(1-\theta)^{-p}\right)^{\mu \cdot(q+p) /(p \cdot q)} \cdot V\left(B_{o}(r)\right)^{-1 / q} \cdot\left(\int_{B_{o}(r)} f^{q}\right)^{1 / q}
$$

for some appropriate constant $C_{1}>0$ depending only on $\mu, p, q, C_{s}$. 


\section{Harnack inequality}

LemMa 3.1. Let $M$ be a complete Riemannian manifold, and a geodesic ball $B_{o}(R)$ satisfies: $\quad B_{o}(R) \cap \partial M=\emptyset$. If it satisfies the following conditions:

(1) For any $0<r<R$, there exist a constant $\eta>0$, such that

$$
V\left(B_{o}(r)\right) \leq 2^{\eta} \cdot V\left(B_{o}\left(\frac{r}{2}\right)\right)
$$

(2) Poincaré inequality, i.e there exist a constant $C_{p}>0$ such that

$$
\int_{B_{o}(r)}\left|f-f_{B}\right|^{p} \leq C_{p} \cdot r^{p} \cdot \int_{B_{o}(r)}|\nabla f|^{p}
$$

for any $0<r<R, f \in H_{1, p}\left(B_{o}(r)\right)$. Where $f_{B}=\int_{B_{o}(r)} f / V\left(B_{o}(r)\right)$.

(3) Sobolev inequality, i.e there exist a constant $C_{s}>0$ such that:

$$
\begin{aligned}
\left(\int_{B_{o}(r)} \phi^{p \mu /(\mu-p)}\right)^{(\mu-p) / p \mu} \leq & C_{s} \cdot V\left(B_{o}(r)\right)^{-1 / \mu} \cdot r \\
& \cdot\left\{\left(\int_{B_{o}(r)}|\nabla \phi|^{p}\right)^{1 / p}+r^{-1} \cdot\left(\int_{B_{o}(r)}|\phi|^{p}\right)^{1 / p}\right\}
\end{aligned}
$$

for any $\phi \in H_{1, p}^{c}\left(B_{o}(r)\right), 0<r<R$. Where constants $\mu>p>1, C_{s}>0$, and $V\left(B_{o}(r)\right)$ denotes the volume of geodesic ball $B_{o}(r)$.

Assuming $f \in H_{1, p}\left(B_{o}(R)\right)$ is a nonnegative function, and satisfies the following inequality in the weak sence

$$
\operatorname{div}\left(|\nabla f|^{p-2} \nabla f\right) \leq A \cdot f^{p-1}
$$

for some constant $A \geq 0$; then for $q>0$ sufficiently small, there must be exist a constant $C_{5}>0$, depending only on $q, \mu, C_{s}, p \eta, C_{p},\left(A R^{p}+1\right)$, such that:

$$
\left\{\frac{\int_{B_{o}(R / 8)} f^{q} d v_{g}}{V\left(B_{o}(R / 8)\right)}\right\}^{1 / q} \leq C_{5} \cdot \inf _{B_{o}(R / 16)} f
$$

Proof. For any $\varepsilon>0$, setting $f_{\varepsilon}=f+\varepsilon, f_{\varepsilon}$ satisfies the inequality (3.4). Letting $\varepsilon \rightarrow 0$, it is sufficient to prove that $f_{\varepsilon}$ satisfies the inequality (3.5). So we can assume that $f \geq \varepsilon>0$, then the function $f^{-1}$ is in $H_{1, p}\left(B_{o}(R)\right)$ and satisfies:

$$
\begin{aligned}
\operatorname{div}\left(\left|\nabla\left(f^{-1}\right)\right|^{p-2} \nabla\left(f^{-1}\right)\right)= & \operatorname{div}\left(-f^{-2(p-1)} \cdot|\nabla f|^{p-2} \cdot \nabla f\right) \\
= & -f^{-2(p-1)} \cdot \operatorname{div}\left(|\nabla f|^{p-2} \cdot \nabla f\right) \\
& +2(p-1) \cdot f^{-2 p+1} \cdot|\nabla f|^{p} \\
\geq & -A \cdot f^{-(p-1)}
\end{aligned}
$$


Applying proposition 2.2 , there exist a constant $C_{2}^{\prime}>0$, depending only on $q, p$, $\mu, C_{s}$ such that:

$$
\begin{aligned}
\left(\inf _{B_{o}(R / 16)} f\right)^{-1} & =\sup _{B_{o}(R / 16)} f^{-1} \\
& \leq C_{2}^{\prime} \cdot\left(A R^{p}+1\right)^{\mu(p+q) /(p \cdot q)} \cdot\left\{\frac{\int_{B_{o}(R / 8)} f^{-q} d v_{g}}{V\left(B_{o}(R / 8)\right)}\right\}^{1 / q}
\end{aligned}
$$

Clearly, the lemma follows if we can estimate the product

$$
\left\{\frac{\int_{B_{o}(R / 8)} f^{q} d v_{g}}{V\left(B_{o}(R / 8)\right)}\right\}^{1 / q} \cdot\left\{\frac{\int_{B_{o}(R / 8)} f^{-q} d v_{g}}{V\left(B_{o}(R / 8)\right)}\right\}^{1 / q}
$$

from above for some value of $q>0$.

To achieve this, let us consider the function $u=\beta+\log f$, where $\beta=$ $-\int_{B_{o}(R / 2)} \log f d v_{g}$; then $u$ satisfies:

$$
\begin{aligned}
\operatorname{div}\left(|\nabla u|^{p-2} \nabla u\right) & =\operatorname{div}\left(f^{-(p-1)} \cdot|\nabla f|^{p-2} \cdot \nabla f\right) \\
& =f^{-(p-1)} \cdot \operatorname{div}\left(|\nabla f|^{p-2} \cdot \nabla f\right)-(p-1) f^{-p} \cdot|\nabla f|^{p} \\
& \leq A-(p-1) \cdot|\nabla u|^{p}
\end{aligned}
$$

Let $\psi$ the cut-off function defined by:

$$
\psi(x)= \begin{cases}0, & \text { for } x \in M \backslash B_{o}(R) \\ \frac{2(R-r(x))}{R}, & \text { for } x \in B_{o}(R) \backslash B_{o}\left(\frac{R}{2}\right) \\ 1, & \text { for } x \in B_{o}\left(\frac{R}{2}\right)\end{cases}
$$

where $r(x)$ is the distance from $o$ to $x$.

Multiplying (3.7) by $\psi^{p}$ and integrating, we have:

$$
\begin{aligned}
(p-1) \cdot \int|\nabla u|^{p} \cdot \psi^{p} & \leq A \int \psi^{p}-\int \psi^{p} \cdot \operatorname{div}\left(|\nabla u|^{p-2} \nabla u\right) \\
& \leq A \int \psi^{p}+p \int|\nabla u|^{p-1} \cdot \psi^{p-1} \cdot|\nabla \psi| \\
& \leq A \int \psi^{p}+\frac{p-1}{2} \int \psi^{p} \cdot|\nabla u|^{p}+2^{p-1} \cdot \int|\nabla \psi|^{p}
\end{aligned}
$$

where we have used Green's formula, Schwartz inequality, and Young inequality. by the above ineguality, we have 


$$
\begin{aligned}
\int_{B_{o}(R / 2)}|\nabla u|^{p} & \leq \int \psi^{p} \cdot|\nabla u|^{p} \\
& \leq \frac{2 A}{p-1} \int \psi^{p}+\frac{2^{p}}{p-1} \int|\nabla \psi|^{p} \\
& \leq \frac{4^{p}}{p-1} \cdot\left(A R^{p}+1\right) \cdot \frac{V\left(B_{o}(R)\right)}{R^{p}}
\end{aligned}
$$

the Poincare inequality (3.2) and (3.9) implies that:

$$
\begin{aligned}
\int_{B_{o}(R / 2)}|u|^{p} & \leq \frac{C_{p} \cdot R^{p}}{2^{p}} \int_{B_{o}(R / 2)}|\nabla u|^{p} \\
& \leq C_{6} \cdot V\left(B_{o}(R)\right)
\end{aligned}
$$

denoted $C_{6}=2^{p} \cdot C_{p} /(p-1) \cdot\left(A R^{p}+1\right)$.

For $\forall q \leq p$, using Hölder inequality, we have

$$
\begin{aligned}
\int_{B_{o}(R / 2)}|u|^{q} & \leq\left(\int_{B_{o}(R / 2)}|u|^{p}\right)^{q / p} \cdot\left(\int_{B_{o}(R / 2)} 1\right)^{1-(q / p)} \\
& \leq C_{6}^{q / p} \cdot V\left(B_{o}(R)\right)
\end{aligned}
$$

On the other hand, let $\phi$ be a Lipschitz cut-off function, given by

$$
\phi(x)= \begin{cases}0, & \text { for } x \in M \backslash B_{o}(\rho+\sigma) \\ \frac{\rho+\sigma-r(x)}{\sigma}, & \text { for } x \in B_{o}(\rho+\sigma) \backslash B_{o}(\rho) \\ 1, & \text { for } x \in B_{o}(\rho) .\end{cases}
$$

where $\rho, \sigma>0, \rho+\sigma \leq R$.

Then multiplying $\phi^{p} \cdot|u|^{p a-p}$ to (3.7) for $a \geq 2$, and integrating by parts yields

$$
\begin{aligned}
(p-1) & \int \phi^{p} \cdot|u|^{p a-p} \cdot|\nabla u|^{p} \\
\leq & A \int \phi^{p} \cdot|u|^{p a-p}-\int \operatorname{div}\left(|\nabla u|^{p-2} \nabla u\right) \cdot \phi^{p} \cdot|u|^{p a-p} \\
\leq & A \int \phi^{p}|u|^{p a-p}+(p a-p) \cdot \int \phi^{p} \cdot|u|^{p a-p-1} \cdot|\nabla u|^{p} \\
& +p \cdot \int|\nabla u|^{p-2} \cdot \phi^{p-1} \cdot|u|^{p a-p} \cdot\langle\nabla u, \nabla \phi\rangle
\end{aligned}
$$

by Young inequality we have:

$$
\begin{aligned}
& (p a-p) \cdot \phi^{p} \cdot|u|^{p a-p-1} \cdot|\nabla u|^{p} \\
& \quad \leq \frac{p-1}{4} \cdot|u|^{p a-p} \cdot \phi^{p} \cdot|\nabla u|^{p}+\left(\frac{4(p a-p-1)}{p-1}\right)^{p a-p-1} \cdot \phi^{p} \cdot|\nabla u|^{p}
\end{aligned}
$$


and

$$
\begin{aligned}
& p \cdot|\nabla u|^{p-2} \cdot \phi^{p-1} \cdot|u|^{p a-p} \cdot\langle\nabla u, \nabla \phi\rangle \\
& \quad \leq \frac{p-1}{4}|u|^{p a-p} \cdot \phi^{p} \cdot|\nabla u|^{p}+4^{p-1} \cdot|\nabla u|^{p a-p} \cdot|\nabla \phi|^{p}
\end{aligned}
$$

Using (3.12), (3.13), (3.14); then

$$
\begin{aligned}
& \int \phi^{p} \cdot|u|^{p a-p} \cdot|\nabla u|^{p} \\
& \leq \frac{2 A}{p-1} \int \phi^{p} \cdot|u|^{p a-p}+\frac{2}{p-1} \cdot\left(\frac{4(p a-p-1)}{p-1}\right)^{p a-p-1} \cdot \int \phi^{p} \cdot|\nabla u|^{p} \\
&+\frac{2 \cdot 4^{p-1}}{p-1} \cdot \int|u|^{p a-p} \cdot|\nabla \phi|^{p} \\
& \leq\left(\frac{2 A}{p-1}+\frac{2 \cdot 4^{p-1}}{(p-1) \cdot \sigma^{p}}\right) \cdot \int_{B_{o}(\rho+\sigma)}^{|u|^{p a-p}} \\
&+\frac{2}{p-1} \cdot\left(\frac{4(p a-p-1)}{p-1}\right)^{p a-p-1} \cdot \int_{B_{o}(\rho+\sigma)}|\nabla u|^{p}
\end{aligned}
$$

By setting $a=2, \rho=R / 4, \sigma=R / 4$; (3.15) becomes:

$$
\int_{B_{o}(R / 4)}|u|^{p} \cdot|\nabla u|^{p} \leq\left(\frac{2 A}{p-1}+\frac{2 \cdot 4^{2 p-1}}{(p-1) \cdot R^{p}}\right) \cdot \int_{B_{o}(R / 2)}|u|^{p}+\frac{2 \cdot 4^{p-1}}{p-1} \cdot \int_{B_{o}(R / 2)}|\nabla u|^{p}
$$

Using (3.9), (3.10), and the last inequality; then:

$$
\int_{B_{o}(R / 4)}|u|^{p} \cdot|\nabla u|^{p} \leq C_{7} \cdot \frac{V\left(B_{o}(R)\right)}{R^{p}}
$$

where we denoted $C_{7}=2 \cdot 4^{2 p-1} /(p-1) \cdot\left(C_{6}+1 /(p-1)\right) \cdot\left(A R^{p}+1\right)$.

Then, we want to estimate $\int_{B_{o}(R / 4)}|u|^{2}$ from above.

(1) When $1<p<2$, for any $\tilde{q} \leq p$, by Hölder inequality, (3.9), and (3.16), we have:

$$
\begin{aligned}
\int_{B_{o}(R / 4)}|u|^{\tilde{q}} \cdot|\nabla u|^{p} & \leq\left(\int_{B_{o}(R / 4)}|u|^{p} \cdot|\nabla u|^{p}\right)^{\tilde{q} / p} \cdot\left(\int_{B_{o}(R / 4)}|\nabla u|^{p}\right)^{1-(\tilde{q} / p)} \\
& \leq C_{7}^{\tilde{q} / p} \cdot\left(\frac{4^{p}}{p-1} \cdot\left(A R^{p}+1\right)\right)^{1-(\tilde{q} / p)} \cdot \frac{V\left(B_{o}(R)\right)}{R^{p}}
\end{aligned}
$$

Let $l \in Z^{+}$, such that $p^{l-1}<2 \leq p^{l}$; and let $1 \leq i \leq l-1$, by Minkowski inequality and Poincare inequality (3.2), then 


$$
\begin{aligned}
\int_{B_{o}(R / 4)} & p^{p p} \cdot|u|^{\left(p^{i}-1\right) \cdot p} \cdot|\nabla u|^{p} \geq \int_{B_{o}(R / 4)}\left|\nabla\left(|u|^{p^{\prime}}\right)\right|^{p} \\
& \geq \frac{4^{p}}{C_{p} \cdot R^{p}} \cdot \int_{B_{o}(R / 4)}\left|\left(|u|^{p^{i}}-V\left(B_{o}\left(\frac{R}{4}\right)\right)^{-1} \cdot \int_{B_{o}(R / 4)}|u|^{p^{l}}\right)\right|^{p} \\
& \geq \frac{4^{p}}{C_{p} \cdot R^{p}}\left[\left(\int_{B_{o}(R / 4)}|u|^{p^{i+1}}\right)^{1 / p}-V\left(B_{o}\left(\frac{R}{4}\right)\right)^{-1+(1 / p)} \cdot \int_{B_{o}(R / 4)}|u|^{p^{p}}\right]^{p}
\end{aligned}
$$

By Hölder inequality, it is easy to show that $\left(\int_{B_{o}(R / 4)}|u|^{p^{1+1}}\right)^{1 / p} \geq$ $V\left(B_{o}(R / 4)\right)^{-1+(1 / p)} \cdot \int_{B_{o}(R / 4)}|u|^{p^{i}}$. then the last inequality becomes:

$$
\begin{aligned}
\left(\int_{B_{o}(R / 4)}|u|^{p^{i+1}}\right)^{1 / p} \leq & \left\{\frac{p^{l p} \cdot C_{p} \cdot R^{p}}{4^{p}} \cdot \int_{B_{o}(R / 4)}|u|^{\left(p^{i}-1\right) \cdot p} \cdot|\nabla u|^{p}\right\}^{1 / p} \\
& +V\left(B_{o}\left(\frac{R}{4}\right)\right)^{-1+(1 / p)} \cdot \int_{B_{o}(R / 4)}|u|^{p^{1}}
\end{aligned}
$$

Using (3.17) and the last inequality, we have:

$$
\begin{aligned}
& \left(\int_{B_{o}(R / 4)}|u|^{p^{i+1}}\right)^{1 / p} \\
& \quad \leq\left\{\frac{p^{l p} \cdot C_{p}}{4^{p}} \cdot C_{7}^{p^{i}-1} \cdot\left[\frac{4^{p}}{p-1} \cdot\left(A R^{p}+1\right)\right]^{p^{l}} \cdot V\left(B_{o}(R)\right)\right\}^{1 / p} \\
& \quad+4^{\eta \cdot(1-(1 / p))} \cdot V\left(B_{o}(R)\right)^{-1+(1 / p)} \cdot \int_{B_{o}(R / 4)}|u|^{p^{l}}
\end{aligned}
$$

Where we have used the condition (1) $V\left(B_{o}(r)\right) \leq 2^{\eta} \cdot V\left(B_{o}(r / 2)\right), 0<r \leq R$. By formula (3.10), one can conclude that: $\int_{B_{o}(R / 4)}|u|^{p} \leq \int_{B_{o}(R / 2)}|u|^{p} \leq C_{6}$. $V\left(B_{o}(R)\right)$. Iterating the inequality $(3.18)$ by finite times, one can conclude that there must be exist a constant $C_{8}>0$, depending only on $p, \eta, C_{p},\left(A R^{p}+1\right)$, such that:

$$
\int_{B_{o}(R / 4)}|u|^{p^{l}} \leq C_{8} \cdot V\left(B_{o}(R)\right)
$$

By Hölder inequality, we have:

$$
\begin{aligned}
\int_{B_{o}(R / 4)}|u|^{2} & \leq\left(\int_{B_{o}(R / 4)}|u|^{p^{l}}\right)^{2 / p^{l}} \cdot\left(\int_{B_{o}(R / 4)} 1\right)^{1-\left(2 / p^{l}\right)} \\
& \leq C_{8}^{2 / p^{l}} \cdot V\left(B_{o}(R)\right)
\end{aligned}
$$


(2) When $p \geq 2$, we have:

$$
\begin{aligned}
\int_{B_{o}(R / 4)}|u|^{2} & \leq\left(\int_{B_{o}(R / 4)}|u|^{p}\right)^{2 / p} \cdot\left(\int_{B_{o}(R / 4)} 1\right)^{1-(2 / p)} \\
& \leq C_{6}^{2 / p} \cdot V\left(B_{o}(R)\right)
\end{aligned}
$$

In any event, (3.19), (3.20) imply that, for any $p>1$, we have the inequality

$$
\int_{B_{o}(R / 4)}|u|^{2} \leq C_{9} \cdot V\left(B_{o}(R)\right)
$$

for some appropriate constant $C_{9}>0$, depending only on $p, \eta, C_{p},\left(A R^{p}+1\right)$.

On the other hand, using the Minkowski inequality and the poincare inequality (3.2), we have:

$$
\begin{aligned}
\int_{B_{o}(R / 4)} & |u|^{p} \cdot|\nabla u|^{p}=\frac{1}{2^{p}} \int_{B_{o}(R / 4)}\left|\nabla\left(u^{2}\right)\right|^{p} \\
\geq & \frac{2^{p}}{C_{p} \cdot R^{p}} \cdot \int_{B_{o}(R / 4)}\left|\left(u^{2}-V\left(B_{o}\left(\frac{R}{4}\right)\right)^{-1} \cdot \int_{B_{o}(R / 4)} u^{2}\right)\right|^{p} \\
\geq & \frac{2^{p}}{C_{p} \cdot R^{p}}\left[\left(\int_{B_{o}(R / 4)} u^{2 p}\right)^{1 / p}-V\left(B_{o}\left(\frac{R}{4}\right)\right)^{-1+(1 / p)} \cdot \int_{B_{o}(R / 4)} u^{2}\right]^{p}
\end{aligned}
$$

by (3.16), (3.21), then (3.22) becomes:

$$
\int_{B_{o}(R / 4)} u^{2 p} \leq C_{10} \cdot V\left(B_{o}(R)\right)
$$

where we denoted: $C_{10}=\left(\left(C_{7} \cdot C_{p} / 2^{p}\right)^{1 / p}+4^{\eta(1-(1 / p))} \cdot C_{9}\right)^{p}$.

For any $\tilde{q} \leq 2 p$, using Hölder inequality one can conclude:

$$
\begin{aligned}
\int_{B_{o}(R / 4)}|u|^{\tilde{q}} & \leq\left(\int_{B_{o}(R / 4)}|u|^{2 p}\right)^{\tilde{q} / 2 p} \cdot\left(\int_{B_{o}(R / 4)} 1\right)^{1-(\tilde{q} / 2 p)} \\
& \leq C_{10}^{\tilde{q} / 2 p} \cdot V\left(B_{o}(R)\right)
\end{aligned}
$$

Let $a \geq 2$, by Cauchy-Schwartz inequality, we have

$$
\left|\nabla\left(\phi|u|^{a}\right)\right|^{p} \leq 2^{p}\left[|\nabla \phi|^{p} \cdot|u|^{a p}+a^{p}|u|^{p a-p} \phi^{p}|\nabla u|^{p}\right]
$$

By the Sobolev inequality, one can conclude: 
338

XI BHANG

$$
\begin{aligned}
& \left(\int_{B_{o}(\rho)}|u|^{a \cdot p \cdot \mu /(\mu-p)}\right)^{(\mu-p) / \mu}\left(\int_{B_{o}(\rho)}\left(\phi|u|^{a}\right)^{p \cdot \mu /(\mu-p)}\right)^{(\mu-p) / \mu} \\
& \quad \leq\left\{C_{s} \cdot V\left(B_{o}(R)\right)^{-1 / \mu}\left[R\left(\int_{B_{o}(R)}\left|\nabla\left(\phi|u|^{a}\right)\right|^{p}\right)^{1 / p}+\left(\int_{B_{o}(R)} \phi^{p}|u|^{a p}\right)^{1 / p}\right]\right\}^{p} \\
& \leq 2^{p} \cdot C_{s}^{p} \cdot V\left(B_{o}(R)\right)^{-p / \mu}\left[R^{p} \int_{B_{o}(R)}\left|\nabla\left(\phi|u|^{a}\right)\right|^{p}+\int_{B_{o}(R)} \phi^{p}|u|^{a p}\right] \\
& \leq 2^{p} \cdot C_{s}^{p} \cdot V\left(B_{o}(R)\right)^{-p / \mu}\left[R^{p} \cdot 2^{p} \cdot a^{p} \int_{B_{o}(R)} \phi^{p} \cdot|u|^{p a-p}|\nabla u|^{p}\right. \\
& \left.\quad+R^{p} \cdot 2^{p} \int_{B_{o}(R)}|u|^{a p} \cdot|\nabla \phi|^{p}+\int_{B_{o}(R)} \phi^{p}|u|^{a p}\right]
\end{aligned}
$$

Using (3.9), (3.15), and the last inequality, we have:

$$
\begin{aligned}
& \left(\int_{B_{o}(\rho)}|u|^{a \cdot p \cdot k}\right)^{1 / k} \\
& \leq 2 \cdot C_{11} \cdot V\left(B_{o}(R)\right)^{(1-k) / k}\left[a^{p} \cdot\left(A R^{p}+\frac{R^{p}}{\sigma^{p}}\right) \int_{B_{o}(\rho+\sigma)}|u|^{p a-p}\right. \\
& \quad+a^{p}\left(\frac{4(p a-p-1)}{p-1}\right)^{p a-p-1} \cdot\left(A R^{p}+1\right) \cdot V\left(B_{o}(R)\right) \\
& \left.\quad+\frac{R^{p}}{\sigma^{p}} \cdot V\left(B_{o}(R)\right)^{-1} \cdot \int_{B_{o}(\rho+\sigma)}|u|^{p a}\right]
\end{aligned}
$$

where we denoted: $C_{11}=2^{P} \cdot C_{s}^{p} \cdot \max \left\{2^{3 p-1} /(p-1), 2^{3 p+1} /(p-1)^{2}, 2^{p+1}\right\}, k=$ $\mu /(\mu-p)$.

It is easy to show that: $|u|^{p a-p} \leq|u|^{p a}+1$, and let $\rho \geq R / 8$, then (3.26) becomes:

$$
\begin{aligned}
& \left(V\left(B_{o}(\rho)\right)^{-1} \int_{B_{o}(\rho)}|u|^{a \cdot p \cdot k}\right)^{1 /(k \cdot p \cdot a)} \\
& \leq\left(8^{\eta} \cdot V\left(B_{o}(R)\right)^{-1} \int_{B_{o}(\rho)}|u|^{a \cdot p \cdot k}\right)^{1 /(k \cdot p \cdot a)} \\
& \leq \\
& \quad\left(2 \cdot 8^{\eta / k} \cdot C_{11}\right)^{1 / p a} \cdot\left[a^{p} \cdot\left(A R^{p}+\frac{R^{p}}{\sigma^{p}}\right) V\left(B_{o}(R)\right)^{-1} \cdot \int_{B_{o}(\rho+\sigma)}|u|^{p a}\right. \\
& \left.\quad+a^{p+p a}\left(\frac{4 p}{p-1}\right)^{p a} \cdot\left(A R^{p}+1\right)\right]^{1 / p a}
\end{aligned}
$$




$$
\begin{aligned}
\leq & C_{12}^{1 / p a} \cdot a^{1 / a} \cdot\left(A R^{p}+\frac{R^{p}}{\sigma^{p}}\right)^{1 / p a} \cdot\left(V\left(B_{o}(R)\right)^{-1} \cdot \int_{B_{o}(\rho+\sigma)}|u|^{p a}\right)^{1 / p a} \\
& +C_{12}^{1 / p a} a^{1+(1 / a)}\left(\frac{4 p}{p-1}\right) \cdot\left(A R^{p}+1\right)^{1 / p a}
\end{aligned}
$$

where $C_{12}=2 \cdot 8^{\eta / k} \cdot C_{11}$.

Let: $a_{i}=2 k^{l}, \sigma_{l}=2^{-4-\imath}, \rho_{i}=R / 4-\sum_{J=0}^{l} \sigma_{J}$, for $i=0,1,2, \ldots ; \rho_{-1}=R / 4$. applying (3.27) to $a=a_{i}, \rho=\rho_{i}, \sigma=\sigma_{l}$; then

$$
\begin{aligned}
\left(V\left(B_{o}\left(\rho_{i}\right)\right)^{-1} \cdot \int_{B_{o}\left(\rho_{l}\right)}|u|^{2 p k^{i+1}}\right)^{1 /\left(2 p k^{t+1}\right)} \\
\leq C_{13}^{k^{-t}} \cdot D^{i k^{-l}}\left(V\left(B_{o}\left(\rho_{i-1}\right)\right)^{-1} \cdot \int_{B_{o}\left(\rho_{t-1}\right)}|u|^{2 p k^{l}}\right)^{1 / 2 p k^{l}} \\
\quad+C_{13}^{k^{-t}} \cdot D^{i k^{-\imath}} \cdot k^{l} \cdot\left(\frac{8 p}{p-1}\right)
\end{aligned}
$$

where we denoted: $C_{13}=\left(C_{12} \cdot\left(A R^{p}+1\right) \cdot 2^{4+p}\right)^{1 / 2 p}, D=2^{1 / 2 p} \cdot k^{1 / 2}$.

Iterating the inequality (3.28), we have:

$$
\begin{aligned}
&\left(V\left(B_{o}\left(\rho_{l}\right)\right)^{-1} \cdot \int_{B_{o}\left(\rho_{l}\right)}|u|^{2 p k^{l+1}}\right)^{1 /\left(2 p k^{l+1}\right)} \\
& \leq \prod_{l=0}^{l} C_{13}^{k^{-l}} \cdot D^{i k^{-l}} \cdot\left(V\left(B_{o}\left(\frac{R}{4}\right)\right)^{-1} \cdot \int_{B_{o}(R / 4)}|u|^{2 p}\right)^{1 / 2 p} \\
&+\left(\frac{8 p}{p-1}\right) \sum_{l=0}^{l-1} C_{13}^{k^{-l}} \cdot D^{i k^{-l}} \cdot k^{l} \cdot \prod_{J=l+1}^{l}\left(C_{13}^{k^{-l}} \cdot D^{i k^{-l}}\right) \\
&+C_{13}^{k^{-l}} \cdot K^{l} \cdot D^{l k^{-l}} \cdot\left(\frac{8 p}{p-1}\right) \\
& \leq C_{14}\left(\left(V\left(B_{o}\left(\frac{R}{4}\right)\right)^{-1} \cdot \int_{B_{o}(R / 4)}^{|u|^{2 p}}\right)^{1 / 2 p}+\sum_{l=0}^{l} k^{l}\right) \\
& \leq C_{14}\left(\left(V\left(B_{o}\left(\frac{R}{4}\right)\right)^{-1} \cdot \int_{B_{o}(R / 4)}|u|^{2 p}\right)^{1 / 2 p}+\frac{\mu}{p} \cdot k^{l}\right)
\end{aligned}
$$

where $C_{14}=(8 p /(p-1)) \prod_{l=0}^{\infty}\left(C_{13}+1\right) k^{-\imath} \cdot D^{i k^{-t}}$. 
For any $j>2 p$; let $l \in N$ such that: $2 p k^{l}<j \leq 2 p k^{l+1}$, then

$$
\begin{aligned}
& V\left(B_{o}\left(\frac{R}{8}\right)\right)^{-1} \cdot \int_{B_{o}(R / 8)}|u|^{J} \\
& \leq\left\{V\left(B_{o}\left(\frac{R}{8}\right)\right)^{-1} \cdot \int_{B_{o}(R / 8)}|u|^{2 p k^{l+1}}\right\}^{J /\left(2 p k^{l+1}\right)} \\
& \leq\left\{2^{\eta} \cdot V\left(B_{o}\left(\rho_{l}\right)\right)^{-1} \cdot \int_{B_{o}\left(\rho_{l}\right)}|u|^{2 p k^{l+1}}\right\}^{J /\left(2 p k^{l+1}\right)} \\
& \leq\left\{2^{\eta} \cdot C_{14}\left(\left(V\left(B_{o}\left(\frac{R}{4}\right)\right)^{-1} \cdot \int_{B_{o}(R / 4)}|u|^{2 p}\right)^{1 / 2 p}+\frac{\mu}{p} \cdot k^{l}\right)\right\}^{J} \\
& \leq C_{15}^{J} \cdot\left(\left(V\left(B_{o}\left(\frac{R}{4}\right)\right)^{-1} \cdot \int_{B_{o}(R / 4)}|u|^{2 p}\right)^{1 / 2 p}+j\right)^{J}
\end{aligned}
$$

where $C_{15}=2^{\eta} \cdot C_{14} \cdot\left(\mu /\left(2 p^{2}\right)+1\right)$.

By (3.24), (3.30) we have:

$$
\begin{aligned}
V\left(B_{o}\left(\frac{R}{8}\right)\right)^{-1} \cdot \int_{B_{o}(R / 8)} e^{q \cdot|u|} & =\sum_{j=0}^{\infty}(j !)^{-1} \cdot q^{J} \cdot V\left(B_{o}\left(\frac{R}{8}\right)\right)^{-1} \int_{B_{o}(R / 8)}|u|^{J} \\
& \leq C_{16}+\sum_{j>2 p}^{\infty}(j !)^{-1} \cdot\left(C_{17} q \cdot j\right)^{j}
\end{aligned}
$$

where $C_{16}, C_{17}$ is appropriate positive constantes depending only on $C_{p}, C_{s}, \eta, p$, $\mu, A R^{p}+1$. By the Stirling inequality, we have:

$$
j^{\jmath} \leq(j !) \cdot e^{\jmath}
$$

then, (3.31) becomes:

$$
V\left(B_{o}\left(\frac{R}{8}\right)\right)^{-1} \cdot \int_{B_{o}(R / 8)} e^{q \cdot|u|} \leq C_{16}+\sum_{j>2 p}^{\infty}\left(C_{17} \cdot q \cdot e\right)^{J}
$$

Let $q \leq(1 / 2) \cdot\left(C_{17} \cdot e\right)^{-1}$, we have:

$$
V\left(B_{o}\left(\frac{R}{8}\right)\right)^{-1} \cdot \int_{B_{o}(R / 8)} e^{q|u|} \leq C_{18}
$$

where $C_{18}$ is a appropriate positive constant depending only on $C_{p}, C_{s}, \eta, p, \mu$, $A R^{p}+1$. Applying inequalities: $e^{q \beta} \cdot f^{q}=e^{q u} \leq e^{q \cdot|u|}, e^{-q \beta} \cdot f^{-q}=e^{-q u} \leq e^{q \cdot|u|}$; we have: 


$$
\begin{gathered}
\left\{V\left(B_{o}\left(\frac{R}{8}\right)\right)^{-1} \int_{B_{o}(R / 8)} f^{-q}\right\}^{1 / q} \cdot\left\{V\left(B_{o}\left(\frac{R}{8}\right)\right)^{-1} \int_{B_{o}(R / 8)} f^{q}\right\}^{1 / q} \\
\quad \leq\left\{V\left(B_{o}\left(\frac{R}{8}\right)\right)^{-1} \int_{B_{o}(R / 8)} e^{q \cdot|u|}\right\}^{2 / q} \leq C_{18}^{2 / q}
\end{gathered}
$$

When $q \leq(1 / 2)\left(C_{17} \cdot e\right)^{-1}$, by (3.6), (3.34), there exist a positive constant depending only on $C_{p}, C_{s}, \eta, p, q, \mu, A R^{p}+1$; such that:

$$
\left\{V\left(B_{o}\left(\frac{R}{8}\right)\right)^{-1} \int_{B_{o}(R / 8)} f^{q}\right\}^{1 / q} \leq C_{5} \cdot \inf _{B_{o}(R / 16)} f
$$

Combining Proposition 2.2 and Lemma 3.1, we have the following locally Harnack inequality.

THEOREM 3.2. Let $M$ be a complete Riemannian manifold, and geodesic ball $B_{o}(R)$ satisfies: $B_{o}(R) \cap \partial M=\emptyset$. If it satisfies the conditions (1), (2), (3) in Lemma 3.1. Fix $p>1$, assuming $f \in H_{1, p}\left(B_{o}(R)\right)$ is a nonnegative function, and satisfies the following inequality in the distribiution sence,

$$
\left|\operatorname{div}\left(|\nabla f|^{p-2} \nabla f\right)\right| \leq A \cdot f^{p-1}
$$

for some constant $A \geq 0$; then, there must be exist a constant $C_{19}>0$, depending only on $p, \mu, C_{s}, p \eta, C_{p},\left(A R^{p}+1\right)$, such that:

$$
\sup _{B_{o}(R / 16)} f \leq C_{19} \cdot \inf _{B_{o}(R / 16)} f
$$

Remark. When $p=2$, Theorem 3.2 is just the result due to $\mathrm{P}$. $\mathrm{Li}$ in [L]. In the special case $A=0$, by Theorem 3.2, we can conclude a globally Harnack inequality which is similar to a result of M. Rigoli, M. Salvatori, and M. Vignati in [2], then Theorem 3.2 can be seen as a generalization of the result in [2].

Proposition 3.3. Let $M$ be a complete noncompact Riemannian manifold (without boudary), and $o$ be a fixed point in $M$. Assuming for any $R>0$ geodesic ball $B_{o}(R)$ satisfies the conditions (1), (2), (3) in Lemma 3.1. Fix $p>1$, let $f \in H_{1, p}(M)$ is a nonnegative function, and satisfies the following quality in the distribiution sence,

$$
\operatorname{div}\left(|\nabla f|^{p-2} \nabla f\right)=0
$$

then, for any $R>0$, there must be exist a constant $C_{20}>0$, depending only on $p$, $\mu, C_{s}, p \eta, C_{p}$, such that:

$$
\sup _{B_{o}(R)} f \leq C_{20} \cdot \inf _{B_{o}(R)} f
$$

By the above grobally Harnack inequality, one can conclude a Liouville theorem for weakly $p$-harmonic function. 
COROLlaRY 3.4. Let $M$ be a complete noncompact Riemannian manifold (without boundary), and $o$ be a fixed point in $M$. Assuming for any $R>0$ geodesic ball $B_{o}(R)$ satisfies the conditions (1), (2), (3) in Lemma 3.1. Fix $p>1$, let $f$ is a nonnegative weakly p-harmonic function $(p>1)$, then $f$ must be constantly.

By the Gromove-Bishop volume comparision theorem and the results due to Saloff-Coste in [5], the conditions (1), (2), (3) in Lemma 3.1 is guaranteed, in the assumption $\operatorname{Ric}_{M} \geq 0$ on $M$. Then, we have the following Corollary.

COROLlARY 3.5. Let $M$ be a complete noncompact Riemannian manifold with nonnegative Ricci curvature, then there is no non-constantly nonnegative weakly pharmonic function. $\quad(p>1)$

\section{Hölder estimate}

THEOREM 4.1. Let $M$ be a complete Riemannian manifold, and geodesic ball $B_{o}\left(R_{0}\right)$ satisfies: $B_{o}\left(R_{0}\right) \cap \partial M=\emptyset$. If it satisfies the conditions (1), (2), (3) in Lemma (3.1). Fix $p>1$, assuming the $u \in H_{1, p}\left(B_{o}\left(R_{0}\right)\right) \cap L^{\infty}\left(B_{o}\left(R_{0}\right)\right)$ and that satisfies the following inequality in the distribiution sence,

$$
\left|\operatorname{div}\left(|\nabla u|^{p-2} \nabla u\right)\right| \leq A
$$

for some constant $A \geq 0$; then, $u$ must be $\alpha$-Hölder contiuous at $o$. and Hölder exponent $\alpha$ depending only on $p, \mu, C_{s}, C_{p}, \eta, C_{p}$.

Proof. Denote: $S(R)=\sup _{B_{o}(R)} u, \quad i(R)=\inf _{B_{o}(R)} u$; let $\quad f=S(R)-u+$ $A^{1 /(p-1)} \cdot R^{p /(p-1)}, g=u-i(R)+A^{1 /(p-1)} \cdot R^{p /(p-1)}$, applying Theorem 3.2 to $f$ and $g$, we have:

$$
\begin{gathered}
S(R)-i\left(\frac{R}{16}\right)+A^{1 /(p-1)} \cdot R^{p /(p-1)} \leq C_{22}\left(S(R)-S\left(\frac{R}{16}\right)+A^{1 /(p-1)} \cdot R^{p /(p-1)}\right) \\
S\left(\frac{R}{16}\right)-i(R)+A^{1 /(p-1)} \cdot R^{p /(p-1)} \leq C_{22}\left(i\left(\frac{R}{16}\right)-i(R)+A^{1 /(p-1)} \cdot R^{p /(p-1)}\right)
\end{gathered}
$$

where $C_{22}$ is a positive constant depending only on $C_{p}, C_{s}, \eta, \mu, p$. Denote: $a=\left(C_{22}-1\right) /\left(C_{22}+1\right)<1, \omega=S(R)-i(R)$, by the above inequalities, we have

$$
\omega\left(\frac{R}{16}\right) \leq a\left(\omega(R)+2 A^{1 /(p-1)} \cdot R^{p /(p-1)}\right)
$$

Iterating (4.2), we have:

$$
\begin{aligned}
\omega\left(16^{-m} \cdot R\right) & \leq a^{m} \cdot \omega(R)+2 A^{1 /(p-1)} \cdot R^{p /(p-1)} \sum_{l=1}^{m} a^{l} \\
& \leq a^{m} \cdot \omega(R)+2 A^{1 /(p-1)} \cdot R^{p /(p-1)} \frac{a}{1-a}
\end{aligned}
$$


For $\forall 0<R<R_{1} \leq R_{0}$, let: $(1 / 16)^{l} R_{1}<R \leq(1 / 16)^{l-1} R_{1}$, by $(4.3)$, we have:

$$
\begin{aligned}
\omega(R) & \leq \omega\left(\left(\frac{1}{16}\right)^{l-1} \cdot R_{1}\right) \\
& \leq a^{l-1} \cdot \omega\left(R_{1}\right)+2 \cdot A^{1 /(p-1)} \cdot R_{1}^{p /(p-1)} \cdot \frac{a}{1-a} \\
& \leq a^{-1} \cdot\left(\frac{R}{R_{1}}\right)^{-\log a / \log 16} \cdot \omega\left(R_{1}\right)+2 \cdot A^{1 /(p-1)} \cdot R_{1}^{p /(p-1)} \cdot \frac{a}{1-a}
\end{aligned}
$$

let $R_{1}=R_{0}^{1-t} \cdot R^{t}, 0<t<1$, then

$$
\begin{aligned}
\omega(R) \leq & a^{-1} \cdot\left(\frac{R}{R_{0}}\right)^{-(1-t) \cdot(\log a / \log 16)} \cdot \omega\left(R_{0}\right) \\
& +2 \cdot A^{1 /(p-1)} \cdot R_{0}^{p /(p-1) \cdot(1-t)} \cdot R^{p /(p-1) \cdot t} \frac{a}{1-a}
\end{aligned}
$$

let $t=(-\log a / \log 16) \cdot(p /(p-1)-\log a / \log 16)^{-1}$, and denote $\alpha=p /(p-1)$. $(-\log a / \log 16) \cdot(p /(p-1)-\log a / \log 16)^{-1}$, by the last inequality, we have:

$$
\omega(R) \leq R^{\alpha} \cdot\left(\frac{\omega\left(R_{0}\right)}{a \cdot R_{0}^{\alpha}}+\frac{2 a A^{1 /(p-1)} \cdot R_{0}^{p /(p-1)-\alpha}}{1-a}\right)
$$

for any $0<R<R_{0}$.

When $A=0$, by inequality (4.5), for any $0<R<R_{0}$, we have:

$$
\omega(R) \leq R^{\alpha} \cdot\left(\frac{\omega\left(R_{0}\right)}{a \cdot R_{0}^{\alpha}}\right)
$$

if $|f(x)|=o\left(\rho^{\alpha}(x)\right)$, as $x \rightarrow 0$, where $\rho(x)$ denotes the geodesic distance from $o$ to $x$; letting $R_{0} \rightarrow 0$, then $f \equiv$ constant. This is the proof of the following theorem.

THEOREM 4.2. Let $M$ be a complete noncompact Riemannian manifold satisfies the conditions (1), (2), (3) in Lemma 3.1. Then there exists a constant $0<\alpha \leq 1$ such that any $p$-harmonic function $f$ defined on $M$ satisfying the growth condition

$$
|f(x)|=\circ\left(\rho^{\alpha}(x)\right)
$$

as $x \rightarrow 0$, where $\rho(x)$ denotes the geodesic distance from o to $x$; must be identically constant.

COROLlaRY 4.3. Let $M$ be a complete noncompact Riemannian manifold with nonnegative Ricci curvature. Then there exists a constant $0<\alpha \leq 1$ such that any $p$-harmonic function $f$ defined on $M$ satisfying the growth condition

$$
|f(x)|=\circ\left(\rho^{\alpha}(x)\right)
$$


as $x \rightarrow 0$, where $\rho(x)$ denotes the geodesic distance from o to $x ;$ Must be identically constant.

\section{REFERENCES}

[1] P LI, Lecture Notes on Geometric Analysis, Lecture Notes Series 6, Research Institute of Mathematics and Global Analysis Research Center, Seoul National University, (1993).

[2] M. Rigoli, M. Salvatori and M. Vignati, A note on $p$-subharmonic functions on Complete manifolds, Manuscripta Math., 99 (1997), 339-359.

[ 3 ] K. UhLenBecK, Reqularity of nonlineare elliptic systems, Acta. Math., 138 (1977), 219-240.

[4] P TOLKSDORFF, Every where regularity for some quasi-linear systems with a lack of ellipticity, Ann. Mat. Pura Appl., 134 (1983), 241-266.

[5] L. SALOFf-Coste, Uniformmly elliptic operators on Riemannian manifolds, J. Differential Geom., 36 (1992), 417-450.

Department of Mathematics

ZHEJIANG UNIVERSITY (XX)

HANGZHOU 310028

P.R. ChINA 